\title{
Synthesis, Characterization and Evaluation of Carboxymethylated Okra Gum as Suspending Agent in Metronidazole Suspension
}

\author{
*ALALOR, CA; OBUNEZIE, DK
}

\author{
Department of Pharmaceutics and Industrial Pharmacy, Faculty of Pharmacy, Delta State University, Abraka, Nigeria \\ *Corresponding AuthorEmail: khritzz@gmail.com,calalor@delsu.edu.ng \\ Tel.: +234-8033239656, +234-8189418861
}

\begin{abstract}
Modification of natural polymers can alter their physicochemical properties and consequently improve functionality. Modification should be undertaken such that the natural polymers do not lose their biological properties. The purpose of this study was to investigate the suspending properties of plain and modified Okra gum in metronidazole suspension. Okra gum was extracted from the pods of Abelmoschus esculentus and modified by Carboxymethylation. Nine (9) batches of metronidazole suspensions were formulated with $0.5 \%, 1 \%$ and $1.5 \%$ of native Okra gum (Batches KB1-KB3), $0.5 \%, 1 \%$ and $1.5 \%$ of tragacanth (KB4-KB6) and $0.5 \%, 1 \%$ and $1.5 \%$ of carboxymethyl-Okra gum (KB7KB9). The formulated suspensions were evaluated using these parameters: sedimentation volume, redispersibility, $\mathrm{pH}$ and viscosity and compared with tragacanth used as standard gum. Experimental results revealed that the swelling index of plain and modified okra gum were 260 and $525 \%$ respectively giving a 2 -fold increase after modification; while the viscosity of plain and modified okra gum were 150 and $15 \mathrm{mPas}$ respectively giving a 10 -fold decrease in viscosity after modification. Suspensions were acidic with $\mathrm{pH}$ range of 3.83-4.76. The suspensions formulated with carboxymethyl-okra gum had better redispersibility profile with lesser redispersion number ranging from 6-12, as against 9-18 for native okra gum and 7-16 for tragacanth. The study demonstrates that both plain and carboxymethyl-okra gum have good suspending properties and compared favorably with the standard gum, tragacanth. However, modification of okra gum by Carboxymethylation, improved functionality in terms of the swelling index and redispersibility which could result in more stable flocculated pharmaceutical suspension systems.
\end{abstract}

\section{DOI: https://dx.doi.org/10.4314/jasem.v24i1.12}

Copyright: Copyright $($ C) 2020 Alalor and Obunezie. This is an open access article distributed under the Creative Commons Attribution License (CCL), which permits unrestricted use, distribution, and reproduction in any medium, provided the original work is properly cited.

Dates: Received: 30 November 2019; Revised: 20 December 2019; Accepted: 23 December 2019

Keywords: Suspension, Carboxymethylation, Okra gum, Metronidazole

The advent of polymeric materials in the field of pharmaceutical technology, have revolutionize drug delivery and opened new horizons into many more untapped possibilities. The quest by pharmaceutical scientists to improve polymer functionality and potential applicability in various areas of drug formulation and delivery has led to the modification of their physical and chemical properties (Deogadeet al., 2012). Natural gums have been modified to overcome certain drawbacks, like uncontrolled rate of hydration, thickening, reduction in viscosity on storage, and susceptibility to microbial contamination. Modification should be undertaken such that the natural polymers do not lose their biological properties. Gums can be modified either by physical or chemical methods (Desai et al., 2006).

Modification by formation of derivatives of natural polymers enhances their physicochemical properties such as hydrophilicity, solubility, swellability, drug release, targeting, stimuli-responsiveness and film forming. A wide variety of functional groups can be attached to natural gums to make them more suitable for controlling the release of drugs from dosage forms. Such derivative formations include carboxymethylation, carbamoylethylation, cyanoethylation, acetylation, deacetylation, phosphorylation, sulfation and esterification (Rana et al., 2011; Deshmukh et al., 2009).

Okra gum, from the pods of Abelmoschus esculentus is one of the natural polysaccharides currently being studied by pharmaceutical scientist, as a hydrophilic polymer in pharmaceutical dosage forms (Zaharuddin et al., 2014). It has advantage over synthetic polymers as it is safe, chemically inert, cheap, easily available, non-irritant, biodegradable, biocompatible, and ecofriendly (Ogaji et al., 2010). Okra gum contains random coil polysaccharides consisting of galactose, rhamnose and galacturonic acid. Indigenous pharmaceutical manufacturers should therefore 
exploit this potential economic source of excellent pharmaceutical excipient (Nasipuri et al., 1996).

The purpose of the present study therefore was to evaluate the effect of native and modified Abelmoschus esculentus (okra) gum on the physicochemical properties of metronidazole suspension.

\section{MATERIALS AND METHODS}

Materials: Metronidazole powder, Sodium hydroxide pellets (Brovie Chemicals, Guangdong Province, China), Monochloroacetic acid (Sigma Aldrich, USA), Benzoic acid (Fooding, China), Tragacanth gum (Krishna Trading company, New Delhi, India), Chloroform water double strength was freshly prepared in the laboratory, Okra gum was extracted from fresh okra pods obtained from a local market in Abraka, Delta State, Nigeria

Extraction of Abelmoschus esculentus gum (okra gum): The fresh fruits of Abelmoschus esculentus were purchased from a local market in Abraka, Delta State. The fruits were cleaned, washed, sliced and crushed using a mortar and pestle in the laboratory. A $150 \mathrm{~g}$ quantity of the blended fruits of Abelmoschus esculentus was weighed into clean plastic containers containing 4 litres of distilled water and stirred. The mixture was then transferred into a cooking pot and boiled with intermittent stirring for 25 minutes, allowed to cool and the mucilage was filtered through a clean white muslin cloth to obtain a viscous filtrate. The viscous filtrate obtained was treated with acetone to precipitate the gum. The gum was dried in a hot air oven at $40^{\circ} \mathrm{C}$; the cake formed was powdered, passed through a sieve of $150 \mu \mathrm{m}$ mesh size and stored in an air-tight container for further use.

Carboxymethylation of Abelmoschus esculentus gum (okra gum): The carboxymethylation of the Abelmoschus esculentus gum was carried out using the method of Saputra et al., (2015) with some modification. A $10 \mathrm{~g}$ sample of native Abelmoschus esculentus gum was mixed with isopropanol in a conical flask. The mixture was stirred for 10 minutes on a magnetic stirrer and $20 \mathrm{ml}$ of $5 \%$ Sodium hydroxide $(\mathrm{NaOH})$ solution was added. The mixture was allowed to stand for $1 \mathrm{hr}$ at $25^{\circ} \mathrm{C}$ for the alkalization process to be completed. Thereafter, $6 \mathrm{~g}$ of monochloroacetic acid was added and the flask was placed in a water bath with temperature maintained at $55^{\circ} \mathrm{C}$ with intermittent stirring for $3.5 \mathrm{hr}$. The $\mathrm{pH}$ of the mixture was adjusted to 5 , filtered and washed with methanol. The derivatized Abelmoschus esculentus gum (carboxymethyl-okra gum) was dried in a hot air oven at $40^{\circ} \mathrm{C}$, powdered and passed through a $150 \mu \mathrm{m}$ mesh size sieve.

Determination of degree of substitution (DS) for carboxymethylated Okra gum: A $0.5 \mathrm{~g}$ powdered sample of the carboxymethyl-okra gum was dissolved in $30 \mathrm{ml}$ of water and then treated with concentrated hydrochloric acid $(\mathrm{HCl})$. The solution was titrated with $0.2 \mathrm{M}$ sodium hydroxide $(\mathrm{NaOH})$ solution and the burette reading was noted. The degree of substitution (DS) was calculated using the equation below (Patel et al., 2018).

$$
D S=\frac{0.162 B}{1-0.58 B}
$$

Where $\quad B=\frac{\text { Voilume of Noor }}{\text { Weight of Sample }}$

Determination of viscosity of gums: A $1 \% \quad \mathrm{w} / \mathrm{v}$ dispersion of the gums were prepared by weighing $1 \mathrm{~g}$ of the gums into a beaker containing $100 \mathrm{ml}$ of distilled water, the gums were mixed thoroughly using a magnetic stirrer until a smooth consistency was achieved. The viscosities of $1 \% \mathrm{w} / \mathrm{v}$ preparations of native okra gum and carboxymethyl-okra gum were determined using the rotational viscometer (NDJ-1, China). The process was carried out using spindle 2 at a speed of 30 revolutions per min.

Determination of the pH of gums: A $1 \% \quad \mathrm{w} / \mathrm{v}$ dispersion of the gums were prepared by weighing $1 \mathrm{~g}$ of the gums into a beaker containing $100 \mathrm{ml}$ of distilled water, the gums were mixed thoroughly using a magnetic stirrer until a smooth consistency was achieved. The $\mathrm{pH}$ of $1 \% \mathrm{w} / \mathrm{v}$ preparations of native okra gum and carboxymethyl-okra gum were determined using a digital $\mathrm{pH}$ meter (Fisher Scientific Inc., UK).

Solubility behavior of gums: The method of Raymond et al., (2009) was adopted with some modifications. 1 $\mathrm{g}$ of the dry powdered samples of the gums were shaken with $10 \mathrm{ml}$ of different solvents (hot distilled water, cold distilled water, ethanol, acetone and chloroform), and allowed to stand for $30 \mathrm{~min}$ after which it was examined macroscopically.

Determination of swelling index of gums: The method of Bowen and Vadino (1984) was adopted with some modification. $1 \mathrm{~g}$ of native okra gum was placed in a $10 \mathrm{ml}$ measuring cylinder and tapped 200 times. The volume occupied by the gum in the cylinder after tapping was recorded as $V_{1}$. Distilled water was added to reach the $10 \mathrm{ml}$ mark in the cylinder and left undisturbed for $24 \mathrm{hrs}$. The new volume occupied by the gum in the cylinder was recorded as $\mathrm{V}_{2}$. The same 
procedure was used in the determination of the swelling index of the carboxymethyl-okra gum. The swelling index (\%) was calculated using the formula:

SweVhing Index $=\frac{V_{2}-V_{1}}{V_{1}} * 100$

Where $V_{1}=$ initial volume of sample, $\nabla_{2}=$ final swelled volume

Determination of loss on drying: A $1 \mathrm{~g}$ quantity of native okra gum was weighed accurately into a preweighed petri-dish and dried in a hot air oven at $105^{\circ} \mathrm{C}$. The weight of the gum was checked at intervals of $10 \mathrm{~min}$, until a constant weight was obtained. The same procedure was repeated for the carboxymethyl-okra gum. The percentage of weight lost by the powder was calculated using the formula below:

Lass on drying $=\frac{W_{1}-W_{2}}{W_{1}} * 100$

Where $W_{1}=$ initial weight of sample, $W_{2}=$ Final weight of sample

Determination of total Ash value of gums: A clean porcelain dish was ignited in a furnace at $500^{\circ} \mathrm{C}$ for 30 min, allowed to cool in a desiccator and weighed. A $0.5 \mathrm{~g}$ quantity of native okra gum was placed in the porcelain dish and weighed. The porcelain dish was placed in the furnace and heated gently first at $300^{\circ} \mathrm{C}$ for $3 \mathrm{hr}$, after which the temperature of the furnace was raised to $500^{\circ} \mathrm{C}$ for $1 \mathrm{hr}$ to turn the charred gum samples to ash white. The porcelain dish was removed from the furnace, allowed to cool and weighed. The same procedure was followed for the determination of total ash value of the carboxymethyl-okra gum. The total ash value was calculated using the formular below:

TAV $=\frac{\text { Weight of ash }}{\text { Weight of polymer }} * 100$

Where TAV is total ash value

Formulation of Metronidazole suspension: Metronidazole suspension was formulated using the formula in Table 1 below. Metronidazole powder $(4 \mathrm{~g})$, native okra gum $(0.5 \mathrm{~g})$ and benzoic acid $(0.1 \mathrm{~g})$ were triturated together and $10 \mathrm{ml}$ of chloroform water double strength was added gradually; while trituration proceeded until a smooth paste was formed. The mixture was transferred into a calibrated dispensing bottle. The mortar was rinsed with distilled water and transferred into the bottle and the preparation was made up to volume with distilled water and shaken vigorously and labeled. The procedure was repeated using $1 \mathrm{~g}$ and $1.5 \mathrm{~g}$ of native okra gum (batches KB1$\mathrm{KB} 3$ ) as the suspending agent; $0.5 \mathrm{~g}, 1 \mathrm{~g}$, and $1.5 \mathrm{~g}$ tragacanth gum (batches KB4- KB6) as the suspending agent; and $0.5 \mathrm{~g}, 1 \mathrm{~g}$ and $1.5 \mathrm{~g}$ carboxymethyl-okra gum (batches KB7- KB9) as the suspending agent.

\begin{tabular}{llllllllll}
\multicolumn{1}{c}{ Table 1: Composition of formulated metronidazole suspensions } \\
\hline & \multicolumn{10}{c}{ Formulation Batches } \\
\cline { 2 - 10 } Ingredients (g) & KB1 & KB2 & KB3 & KB4 & KB5 & KB6 & KB7 & KB8 & KB9 \\
\hline Metronidazole & 4 & 4 & 4 & 4 & 4 & 4 & 4 & 4 & 4 \\
Okra gum (Plain) & 0.5 & 1 & 1.5 & - & - & - & - & - & - \\
Tragacanth gum & - & - & - & 0.5 & 1 & 1.5 & - & - & - \\
Carboxymethyl-Okra gum & - & - & - & - & - & - & 0.5 & 1 & 1.5 \\
Benzoic acid & 0.1 & 0.1 & 0.1 & 0.1 & 0.1 & 0.1 & 0.1 & 0.1 & 0.1 \\
Chloroform water DS (ml) & 10 & 10 & 10 & 10 & 10 & 10 & 10 & 10 & 10 \\
Distilled water to (ml) & 100 & 100 & 100 & 100 & 100 & 100 & 100 & 100 & 100 \\
\hline
\end{tabular}

pH assessment of suspension: A $50 \mathrm{ml}$ volume of the prepared suspension was measured and transferred into a beaker. The $\mathrm{pH}$ of the different batches of the suspension was assessed using a digital $\mathrm{pH}$ meter. The constant $\mathrm{pH}$ reading displayed was recorded.

Viscosity assessment of suspensions: The viscosities of the prepared suspensions (batches KB1-KB9) were determined at $25^{\circ} \mathrm{C}$ using the rotational viscometer (NDJ-1, China). This was carried out by measuring $100 \mathrm{ml}$ from each batch of suspension into a beaker, the spindle (number 2) was inserted into the beaker with the aid of a guard until the suspension covered the spindle to the appropriate mark, and then the viscometer was set at 30 revolutions per min and operated. The viscosity value was read from the display.

Sedimentation volume: The formulated suspensions (batches KB1-KB9) were poured into $100 \mathrm{ml}$ labeled measuring cylinders, stoppered and placed on a vibration-free surface at $25^{\circ} \mathrm{C}$. The suspensions were monitored and the volumes of the sediments obtained were recorded at $24 \mathrm{hrs}$ interval for a period of 2 weeks. The sedimentation volume $\mathrm{F}(\%)$ was then determined using the equation: 
$F=\frac{V_{u}}{V_{0}}=100$

Where $\mathrm{F}=$ sediment volume, $V_{u}=$ ultimate volume of sediment, $V_{0}=$ original volume of suspension

Redispersibility: After recording the sedimentation volume for 2 weeks, the measuring cylinders containing suspensions from batches KB1-KB9 were stoppered and reconstituted by redispersing the sediments and the ease of redispersibility was measured by the number of cycles of inversions (top to bottom and back to top again) required to completely redisperse suspension without sediments at the bottom. The number of cycles were taken as the redispersibility number for each batch (Uhumwanghoand Ileje, 2014).

\section{RESULTS AND DISCUSSION}

The observed solubility behavior showed that both the plain and modified okra gums were more soluble in hot distilled water, slightly soluble in cold water and insoluble in organic solvents: acetone, ethanol and chloroform. This is due to the fact that gums are polysaccharides with numerous sugar molecules and therefore dissolve in water (Mahmud et al., 2008). The results of the physicochemical characterization of the gums are presented in Table 2

Table 2: Physicochemical properties of Plain Okra gum and

\begin{tabular}{lll}
\multicolumn{2}{c}{ carboxymethyl-okra gum } \\
\cline { 2 - 3 } Parameters & \multicolumn{2}{c}{ Description/ Values } \\
\cline { 2 - 3 } & $\begin{array}{l}\text { Plain } \\
\text { Okra gum }\end{array}$ & $\begin{array}{l}\text { Carboxymethyl- } \\
\text { okra gum }\end{array}$ \\
\hline Viscosity (mPas) & 150 & 15 \\
pH & 5.27 & 6.22 \\
Swelling index (\%) & 260 & 525 \\
Loss on drying (\%) & 0.2554 & 0.4326 \\
Total Ash value (\%) & 4 & 12 \\
\hline
\end{tabular}

The experimental values for total ash content obtained were $4 \%$ and $12 \%$ for the plain okra gum and carboxymethyl-okra gum respectively. These values indicate that the inorganic compounds contained in the gums were low. The values for viscosity obtained showed a 10 -fold decrease in viscosity from 150 to 15 mPas after modification, the plain okra gum was far more viscous than the carboxymethyl-okra gum. Zhao et al., (2003) reported similar behavior for carboxymethylation of cellulose, with a decrease in viscosity after modification. This behavior may be attributed to possible degradation of the original structure of the gum due to the alkali and the etherifying agent.

A swelling index value of $525 \%$ for the carboxymethyl-okra gum indicates its high swelling abilities compared to that of the plain okra-gum with a value of $260 \%$. Swelling of a linear polymer without dissolution is an indication that it is cross-linked. The cross-links tie the macromolecular chains together by primary covalent bonds thereby transforming each particle into a single giant molecule. The swelling ability of polymers enables it to absorb water and this is one of the bases for the major uses of gums.

The $\mathrm{pH}$ of $1 \% \mathrm{w} / \mathrm{v}$ dispersion gave 5.27 and 6.22 for plain okra gum and carboxymethyl-okra gum respectively. This indicates that both gums are weakly acidic. The value for loss on drying gave $0.2554 \%$ and $0.4326 \%$ for the plain okra gum and the carboxymethyl-okra gum respectively. This indicates very low moisture content hence; the gums can be stored for long periods without microbial attack since moisture promotes microbial growth.

Degree of substitution (DS) of carboxymethyl-okra gum: Degree of substitution (DS) is the average number of substituent (functional groups) introduced per anhydroglucose unit (AGU) and varies between 0 and 3 (Rachtanapun et al., 2012); It indicates the amount of substituted carboxymethyl group. The carboxymethylation of the okra gum resulted in a DS of 0.29. The extent of modification by carboxymethylation depends on whether any steric impediments exist in the reactive centers susceptible to etherification and the polysaccharide's anomeric linkage type. The DS value may be improved further by changing the reaction conditions for the carboxymethylation process such as solvent system, solvent composition, concentration of sodium hydroxide and monochloroacetic acid (MCA), reaction time and reaction temperature.

Physical properties of formulated suspensions: The gums were used as suspending agents at concentrations of $0.5,1$ and $1.5 \% \mathrm{w} / \mathrm{v}$ in the formulation of metronidazole suspension and compared with tragacanth gum (used as a standard suspending agent) in a similar formulation. The results for the $\mathrm{pH}$, viscosity and redispersibility are presented in Table 3.

Table 3: pH, Viscosity and Redispersibility of formulated metronidazole suspension

\begin{tabular}{llll}
\hline Batches & pH & $\begin{array}{l}\text { Viscosity } \\
\text { (m.Pas) }\end{array}$ & $\begin{array}{l}\text { Redispersibility } \\
\text { (no. of cycles) }\end{array}$ \\
\hline KB1 & 3.95 & 35 & 9 \\
KB2 & 4.09 & 155 & 12 \\
KB3 & 4.0 & 520 & 18 \\
KB4 & 3.88 & 20 & 7 \\
KB5 & 3.83 & 35 & 10 \\
KB6 & 3.83 & 80 & 16 \\
KB7 & 4.38 & 10 & 6 \\
KB8 & 4.63 & 20 & 8
\end{tabular}




\begin{tabular}{llll} 
KB9 & 4.76 & 35 & 12 \\
\hline
\end{tabular}

The suspensions prepared with the native okra gum (batches KB1-KB3) gave $\mathrm{pH}$ range of 3.95-4.09; the suspensions prepared with tragacanth gum (batches KB4-KB6) had a $\mathrm{pH}$ range of 3.83-3.88 while the suspensions with carboxymethyl-okra gum (batches KB7-KB9) had a $\mathrm{pH}$ range of 4.38-4.76. All formulations can be said to be moderately acidic and this is acceptable since higher antibacterial activity is obtained at acidic $\mathrm{pH}$ levels (Saliani et al., 2015). The viscosity behavior showed that increase in concentration of the different gums in each formulation increased the viscosity of the formulation correspondingly. Suspension prepared with $1.5 \%$ native okra gum was more viscous than suspension prepared with $0.5 \%$ okra gum; same effect was observed in formulations with tragacanth and carboxymethyl-okra gum.

It was also observed that the suspensions formulated with the carboxymethyl-okra gum were less viscous compared to those formulated with tragacanth gum at the same concentration; while those suspensions with native okra gum were more viscous compared to the formulations with tragacanth at the same concentration. Redispersibility test, which indicates how easy it is to redisperse the particles in the suspension, was performed after 2 weeks of storage. The result showed that the formulations with carboxymethyl-okra gum redispersed easily compared to the formulations with tragacanth gum; while it took much agitation for the formulations with the native okra gum to be redispersed.

Sedimentation behavior of formulated suspensions: The sedimentation volumes of the formulations are presented in Figures 1, 2 and 3.The particles in KB1, KB4 and KB7 settled rapidly after formulation. This behavior can be attributed to low concentration of suspending agents $(0.5 \%)$ used in each of the formulation.

The particles in KB2, KB5 and KB8 which contained $1 \%$ of the suspending agent were also observed to settle fast but not as fast as the particles in formulations with $0.5 \%$ of the gums. However, it was observed that the particles in KB3, KB6 and KB9 settled slowly, the particles in these formulations were suspended for longer period of time. This behavior can be attributed to increased concentration (1.5\%) of the suspending agents which minimized the rate of settling of the particles. This property is typical of a well-formulated suspension as it allows for homogeneity from the period between reconstitution and dispensing of the required dose, thereby enhancing proper medication dosing. The sedimentation behavior of the formulations is further illustrated in Figures 1,2 and 3

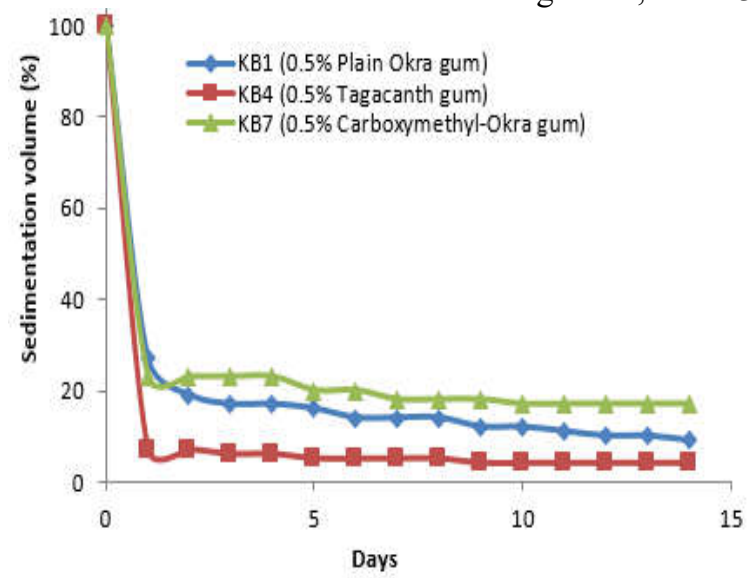

Fig1: Sedimentation volume for $0.5 \% \mathrm{w} / \mathrm{v}$ of the various gums used as suspending agents

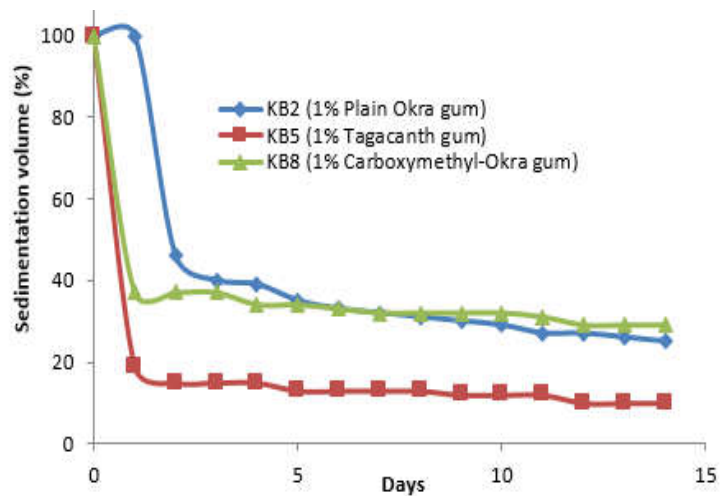

Fig2: Sedimentation volume for $1 \% \mathrm{w} / \mathrm{v}$ of the various gums used as suspending agents

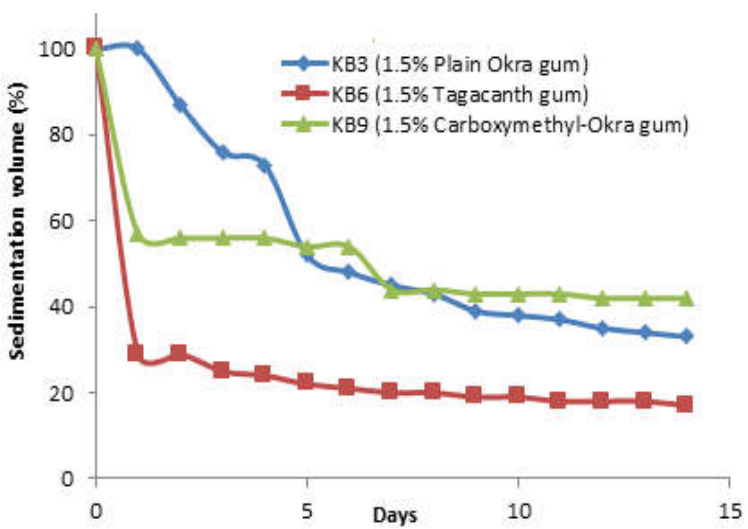

Fig3: Sedimentation volume for $1.5 \% \mathrm{w} / \mathrm{v}$ of the various gums used as suspending agent

Conclusion: Carboxymethyl-okra gum showed improved functionality in term of better swelling capacity than the plain okra gum. This could favour flocculation of the particles in the suspension through formation of loose agglomerates. Metronidazole 
suspensions formulated with carboxymethyl-okra gum showed better redispersibility compared to the other gums used in formulation of metronidazole suspension and as such, will require moderate agitation to redisperse. This will lead to uniformity of dose during administration and further enhance patient therapeutic outcome.

\section{REFERENCES}

Deogade, UM; Deshmukh, VN; Sakarkar, DM (2012). Natural Gums and Mucilage's in NDDS: Applications and Recent approaches. Int. J. PharmTech Res. 4(2): 799-814

Desai, KG; Park, HJ (2006). Study of gamma irradiation: effects on chitosan micropartcles. Drug Deliv. 13:39-50.

Deshmukh, T; Patil, P; Thakare, V; Tekade, B (2011). Evaluation of binding properties of Butea monosperma Lam, gum in tablet formulation, Int. J. Drug Disc. Herbal Res. 1(3):128-133.

Mahmud, HS; Oyi, AR; Allagh, TS (2008). Studies on some physicochemical properties of Khaya senegalensis gum. Nig. J. Pharm. Res. 7(1): 146152.

Nasipuri, RN; Igwilo, CI; Brown, SA; Kunle, OO (1996). Mucilage from Abelmoschus esculentus fruits- a potential pharmaceutical raw material. $J$. Pharm. Res. Dev. 1:22-28.

Ogaji, IJ; Nep, EI; Audu-Peter, JD (2011). Advances in natural polymers as pharmaceutical excipients. Pharm. Anal. Acta. 3(1): 6.

Patel, SH; Baj, YM; Patel, NK (2018). Characterization and Optimization of Reaction Parameters for Sodium Salt of Partially Carboxymethylated Okra Gum. Chem. Sci. Trans. 7(1): 55-62
Rachtanapun, P; Simasatitkul, P; Chaiwan, W; Watthanaworasakun, Y (2012). Effect of sodium hydroxide concentration on properties of carboxymethyl rice starch. Int. Food Res. J. 19(3): 923-931.

Rana, V; Rai, P; Tiwary, AK; Singh, RS; Kennedy, JF; Knill, CJ (2011). Modified gums: Approaches and applications in drug delivery. Carbohydr. Polym. 83(3): 1031-1047

Raymond, CR; Paul, JS (2009). Handbook of Pharmaceutical Excipients $6^{\text {th }}$ Ed; Chicago pharmaceutical press. Pp. 478-479.

Saliani, M; Jalal, R; Kafshdare, E (2015). Effects of $\mathrm{pH}$ and temperature on antibacterial activity of zinc oxide nanofluid against $E$. coli and $S$. aureus. Jundishapur J. Microbiol. 8(2)

Saputra, A.H; Hapsari, M; Pitaloka, AB (2015). Synthesis and characterization of CMC from water hyacinth cellulose using isobutyl-isopropyl alcohol mixture as reaction medium. Contemp. Eng. Sci. 8(33): 1571- 1582

Uhumwangho, MU; Ileje, IL (2014). Preliminary evaluation of the suspending properties of Brachystegia eurycomagum on metronidazole suspension. Int. Curr. Pharm. J. 3(11): 328-330.

Zhao, H; Cheng, F; Li, G; and Zhang, J (2003). Optimization of a process for carboxymethyl cellulose (CMC) preparation in mixed solvents. Int. J.Polym. Mater. 52: 749-759.

Zaharuddin, ND; Noordin, MI; Kadivar, A (2014). The Use of Hibiscus esculentus (Okra) Gum in Sustaining the Release of Propranolol Hydrochloride in a Solid Oral Dosage Form. Biomed. Res. 8 pages. 